\title{
Watching las ruedas fall off
}

\author{
Indiscriminate budget cuts threaten the future of science in Spain.
}

T he great Spanish neuroscientist and Nobel Laureate Santiago Ramon y Cajal once famously said, "Al carro de la cultura española le falta la rueda de la ciencia," which translates as "The carriage of Spanish culture lacks the wheel of science." Beginning in the early 1980s, the Spanish government began the process of reintroducing that wheel to the Spanish economy: over the past 30 years, Spanish research and development productivity has bourgeoned more than tenfold. However, the current government has proposed severe budget cuts that are poised to send the wheel of science spinning off the cart once again. This latest round of slash-and-burn cost-cutting measures indicates a clear lack of foresight on the part of policymakers and threatens to undermine not only the advances made by the Spanish scientific establishment during the past three decades, but also aims to sabotage the research- and technology-based developments that could clearly aid future economic recovery and promote competitiveness in the global research environment.

Last December, the Spanish government put forth a 2012 budget proposal that included a cut in funding for research and development (which includes science research) of nearly $€ 600$ million (a decrease of nearly 7\% from 2011 levels). Since then, this figure has been revised even more dramatically, as the latest budget is slated to cut research funding by over $25 \%$ from 2011 levels, continuing a trend of cuts that began in 2009. What makes these cuts even more egregious is the fact that other ministries are facing an average deficit of only $16 \%$. Adding insult to injury, the government also announced in January that the Ministry of Science and Innovation would be dissolved and folded into the larger Ministry of Economy and Competitiveness, thereby further limiting the ability of those whose job it is to defend the interests of science research to remain involved in budgetary decisions.

It has already become clear that these cuts threaten to undermine the ability of scientific institutes across the country to hire and retain talented personnel. While Spain is emptying the coffers of research and development, other European nations are actually increasing their investment in science. This year, Germany announced a measure to raise the budget of their primary research centers by $5 \%$, while France recently promised a $€ 35$ billion increase in research spending. Drastic funding reductions in Spain will undoubtedly place further limits on the size and resources of academic laboratories, which is likely to result in many established scientists leaving the country for opportunities elsewhere. As the successful and experienced investigators who helped to make Spanish science competitive quit the country, they will leave behind academic holes that simply cannot be filled by their respective institutions.

However, the loss of senior faculty is not the only fallout that these cuts are expected to induce. The Spanish National Research Council (CSIC), Spain's largest public research institution, has been given a budget that will not even cover basic operating costs for the whole of 2012, and some laboratories are considering shutting down for a few weeks over the summer just to stretch their funds. Moreover, current estimates suggest that funds previously destined for programs that cover the employment of new research personnel will be cut by $35.5 \%$, further exacerbating the existing shortage of such staff. For example, the CSIC estimates that they have filled less than $20 \%$ of their minimum personnel requirements for 2010 and 2011, and that number is expected to decrease substantially in 2012. Support for postdoctoral positions have been hit particularly hard, as funding is expected to drop by as much as $43 \%$, while the "Ramon y Cajal" program, which serves as the primary source of tenure-track positions for junior scientists in Spain, will face a similar cut. When considered alongside the proposed freeze on permanent research positions at universities and research centers, the likely outcome is a collective 'brain drain', as the best and brightest trainees flee the sinking Spanish ship for more stable waters elsewhere in Europe or North America or leave science entirely. The end result will almost certainly be a talent vacuum that will require decades to be fully reversed.

In May of 2011, the Spanish Congress and Senate approved the 'Preamble of the Law of Science, Technology and Innovations' by a nearly unanimous vote, agreeing that it was "necessary to promote change through investment in research and innovation as a way to achieve a knowledge-based economy." Several Spanish scientific organizations, including the Spanish Society for Neuroscience, have sent open letters to the Spanish government pointing out that the current indiscriminate budget cuts are in sharp contrast to this promise. Given the severe economic stresses faced by the Spanish government, austerity must undoubtedly be practiced. However, this must be done with careful planning. Implementing funding decisions that are, by its own admission, counter-productive to future economic growth is both irresponsible and dangerous. The current financial trouble is actually a rare opportunity for the Spanish government to start over and take the bold steps that they promised earlier by beginning to lay the groundwork for a research- and innovation-based economy. This can only be achieved by insuring that science does not get discarded by sweeping, undirected budget cuts. A wiser way forward should include policy changes that aim to promote austerity at the level of the local research centers, rewarding efforts that make more efficient use of resources and promoting the development and utilization of shared facilities while providing institutions the financial wherewithal to retain talented scientists and attract the next generation of scientific innovators. The suggestion that Spanish scientists should focus on 'quality over quantity', as posited by Science Secretary Carmen Vela in a recent letter to Nature (http://www.nature.com/news/turn-spains-budget-crisis-into-an-opportunity-1.10770) is missing the point. If Spanish policymakers fail to take the steps necessary to halt this collective brain drain, there will be no talented researchers left in Spain to produce the 'quality' science Dr. Vela envisions, threatening to drive Spanish science into a hole from which it may never fully recover. 